\title{
Preparation of Cellulose Nanocrystals via Successive Periodate and Bisulfite Oxidation and Mechanical and Hydrophilic Properties of the Films
}

\author{
Baoyu Wang, ${ }^{a}$ Rong Li, ${ }^{a}$ Jinhao Zeng, ${ }^{a}$ Min He, ${ }^{a}$ and Junrong Li ${ }^{b}, *$ \\ Microcrystalline cellulose was oxidized via periodate followed by \\ sulfonation. The sulfonated cellulose nanocrystals were obtained through \\ centrifugation, dialysis, and sonication. The sulfonated cellulose \\ nanocrystals were rod-like and had an average length of $140 \mathrm{~nm}$ to 153 \\ $\mathrm{nm}$ and an average width of 8 to $10 \mathrm{~nm}$. The Fourier transform infrared \\ profiles and polyelectrate titration demonstrated successful introduction of \\ the sulfonated groups into the cellulose nanocrystals. The sulfonated \\ cellulose nanocrystals had a higher crystallinity index than dialdehyde \\ cellulose. The thin films fabricated via the casting of the sulfonated \\ cellulose nanocrystals suspensions were highly hydrophilic.
}

Keywords: Sulfonated cellulose; Nanocrystal; Hydrophilicity

Contact information: a: School of Chemical Engineering and Technology, Guangdong Industry

Polytechnic, Guangzhou 510300 China; b: State Key Laboratory of Pulp and Paper Engineering, South China University of Technology, Guangzhou 510640 China; *Corresponding author: lljrr@scut.edu.cn

\section{INTRODUCTION}

Cellulose is the primary component of the cell walls of plants and algae and is the most abundant natural polymer found in nature (Klemm et al. 2005). This bio-based material has low toxicity, it is biocompatible and renewable, and there is a growing interest to replace petrochemical products with cellulose to alleviate environment pollution (Goetz et al. 2009). Nanocellulose refers to cellulose particles having at least one dimension in nanoscale (1 nm to $100 \mathrm{~nm}$ ), which are usually classified as cellulose nanocrystals (CNC) or cellulose nanofibril (CNF). Cellulose nanocrystals are rod-like with a length of $200 \mathrm{~nm}$ to $500 \mathrm{~nm}$ and a diameter of $3 \mathrm{~nm}$ to $35 \mathrm{~nm}$ and can be derived from acidic hydrolysis. However, the acidic hydrolysis process causes problems, e.g., equipment erosion and environmental pollution (Rånby et al. 1949). Cellulose nanofibril particles have a diameter of $5 \mathrm{~nm}$ to $50 \mathrm{~nm}$ and a length of a few micrometers and can be obtained via chemical or enzymatic pretreatment, followed by mechanical treatment. however, mechanical treatment consumes a lot of energy (Nechyporchuk et al. 2016).

It is well known that cellulose can be oxidized with periodate to obtain dialdehyde cellulose (DAC), which occurs when the $\mathrm{C}_{2}-\mathrm{C}_{3}$ bonds are broken and the hydroxyl groups at $\mathrm{C}_{2}$ and $\mathrm{C}_{3}$ are converted into aldehyde groups (Kim et al. 2000). The opening of the $\beta$ D glucose units disrupts the ordered structure of cellulose, and the flexibility of the cellulose chain dramatically increases (Casu et al. 1985). Meanwhile, DAC is a highly active intermediate, which can be further derivatized into dialcohol, dicarboxylate, imine, and sulfonate cellulose (Guigo et al. 2014). In recent years, CNC has been separated via derivative reactions of DAC. Errokh et al. (2018) obtained CNC with a width of $5 \mathrm{~nm}$ to $10 \mathrm{~nm}$ via the $\mathrm{NaBH}_{4}$ reduction of DAC. Yang et al. (2013) separated CNC with a length

Wang et al. (2021). "Sulfonated cellulose nanocrystals," BioResources 16(1), 1713-1725. 1713 
of 120 to $200 \mathrm{~nm}$ and a diameter of approximately $13 \mathrm{~nm}$ via the chlorite oxidation of DAC. Visanko et al. (2014) used a combined procedure of the reductive amination of DAC and its mechanical homogenization to synthesize CNC with both hydrophobic and hydrophilic properties.

The sulfonation of DAC (shown as scheme 1) introduces sulfonated groups into the DAC molecular chains, and the electrostatic force between sulfonated groups acts on the DAC particles, CNC should be obtained via the sulfonation of DAC. However, there are only reports concerning CNF produced via the sulfonation of DAC and the solubility of sulfonated cellulose. Sun et al. (2017) separated CNF via the sulfonation of DAC followed by homogenization, which can be used as an oil/water separator. Pan and Ragauskas (2014) produced CNFs with a width of $15 \mathrm{~nm}$ to $45 \mathrm{~nm}$ and length of $1 \mu \mathrm{m}$ following the same procedure. Thiangtham et al. (2019) obtained transparent sulfonated suspensions via the sulfonation of DAC and found the suspensions contained cellulose particles, but unfortunately there was no further exploration of these particles.

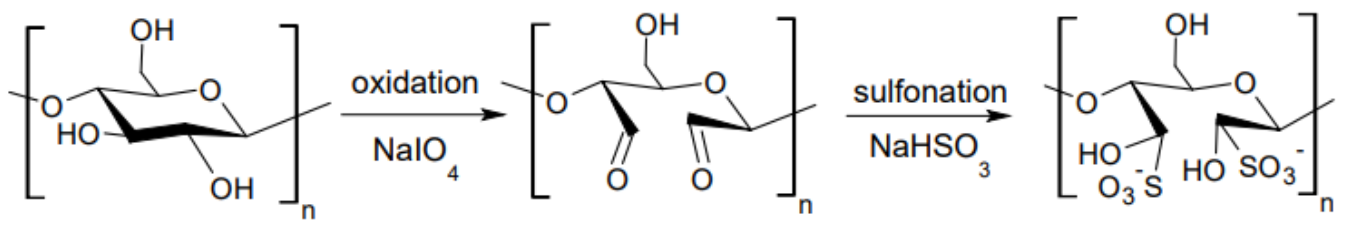

Scheme 1. Sulfonation of DAC

Sulfonated cellulose is a potential immunosorbent material (Rocha et al. 2018) and green flocculation agent for mineral particles (Liimatainen et al. 2013). Sulfonated cellulose film has a potential application as a separator membrane in lithium-ion batteries (Thiangtham et al. 2019). The purpose of this study was to extract sulfonated nanocrystals (SCNCs) via the sulfonation of DAC. Microcrystalline cellulose (MCC) was first oxidized to DAC with a moderate dialdehyde content, followed by sulfonation with sodium bisulfite. Fourier-transform infrared spectroscopy (FTIR), X-ray diffraction (XRD), and atomic force microscopy (AFM) were used to characterize SCNCs. The films were fabricated via the casting of SCNCs suspensions, and the mechanical strength and hydrophilicity of the films were investigated.

\section{EXPERIMENTAL}

\section{Raw Materials}

The MCC (particle size less than $25 \mu \mathrm{m}$ ), sodium periodate, sodium hydroxide, and ammonium hydrochloride were purchased from Aladdin (Shanghai, China). The sodium chlorite, glacial acetic acid, sodium bisulfite, ethylene glycol, and poly (diallyldimethylammonium chloride) (PDADMAC) were purchased from Macklin (Shanghai, China). All the chemicals were of analytical grade or above and used as received. Deionized water was used throughout the experiments.

\section{Extraction of Sulfonate Nanocrystals}

Preparation of DAC

The MCC was oxidized to DAC following the process outlined by Sirviö et al. (2011) with some changes. In summary, $4 \mathrm{~g}$ of MCC, $5.28 \mathrm{~g}$ of $\mathrm{NaIO}_{4}\left(\right.$ molar ratio of $\mathrm{NaIO}_{4}$ 
to $\mathrm{AGU}=1$ to 1 ), $3.364 \mathrm{~g}$ of sodium chloride (molar ratio of $\mathrm{NaCl}$ to $\mathrm{AGU}=7$ to 3 ), and $200 \mathrm{~mL}$ of deionized water were added into a conical flask covered with aluminum foil and the mixture was magnetically stirred in a water bath at $50{ }^{\circ} \mathrm{C}$ for $3 \mathrm{~h}$, followed by the addition of ethylene glycol to terminate the reaction. Subsequently, the suspension was vacuum filtered and washed several times with deionized water until the conductivity was less than $50 \mu \mathrm{S} / \mathrm{cm}$, then rinsed with ethanol. Finally, the oxidized products were vacuum dried and stored for further use.

\section{Sulfonation of DAC}

The sulfonation of DAC was performed according to the following procedure: $2 \mathrm{~g}$ of DAC, $2 \mathrm{~g}$ of sodium bisulfite $(19.2 \mathrm{mM})$, and $200 \mathrm{~mL}$ of deionized water were mixed together and magnetically stirred at room temperature for $6 \mathrm{~h}, 12 \mathrm{~h}$, and $24 \mathrm{~h}$ to obtain the sulfonated cellulose samples correspondingly named $\mathrm{SCNC}_{1}, \mathrm{SCNC}_{2}$, and $\mathrm{SCNC}_{3}$.

\section{Separation of nanocrystals}

The sulfonated cellulose was first centrifuged twice at $10000 \mathrm{rpm}$ for $10 \mathrm{~min}$; the sediment was collected and dialyzed (molecular weight cut-off (MWCO) $=8 \mathrm{kDa}$ to 14 $\mathrm{kDa}$ ) against deionized water until the conductivity was less than $50 \mu \mathrm{S} / \mathrm{cm}$. Subsequently, the dialyzed suspension was sonicated for $10 \mathrm{~min}$ at a power of $650 \mathrm{~W}$ and an amplitude of $80 \%$, and then centrifuged at $10000 \mathrm{rpm}$ for $10 \mathrm{~min}$ to remove any fiber bundles. Finally, the supernatant was collected for further use.

\section{Determination of Aldehyde Group Content}

The aldehyde group content of DAC was determined following the literature procedure (Zhao and Heindel 1991).

\section{Charge Density Measurements}

The charge density of the SCNCs was determined using a particle charge analyzer (PCD-05, BTG Instruments, Värmland, Sweden). The suspension of the sulfonated cellulose was diluted 10 -fold, and $10 \mathrm{~mL}$ of the diluted suspension was pipetted into the measurement cell and then titrated with $0.001 \mathrm{~N}$ of PDADMAC.

\section{Characterization of the Sulfonated Cellulose Nanocrystals (SCNCs)}

The FTIR measurements were carried out with a FTIR spectrometer (Thermo Scientific Nicolet 6700, Waltham, MA); the spectra of the samples were obtained via $\mathrm{KBr}$ pellets in transmission mode. The XRD measurements were performed with an X-ray fluorescence spectrometer (AXIOS-PW4400, Malvern Panalytical, Malvern, United Kingdom) using $\mathrm{Cu} \mathrm{K} \alpha(\lambda=1.5406 \mathrm{~nm})$ radiation. The crystalline index $(\mathrm{CI})$ was evaluated based on the Segal method (Segal et al. 1959).

The morphology of the SCNCs were investigated with an atomic force microscope (AFM) (Nanoscope IIIa, Veeco, Plainview, NY) with silicon cantilever probes in tapping mode; the images were analyzed with Nanoscope Analysis software (version 1.7, Bruker, Billerica, MA). The dimensions of the SCNCs were determined with a nanoparticle analyzer (SZ-100, Horiba, Kyoto, Japan). The scattering angle was $90^{\circ}$ and six tests were conducted for each sample. The Z-average diameter and polydispersity index were averaged for each sample. 


\section{Mechanical Strength of the Sulfonated Cellulose Nanocrystals (SCNCs) Films}

The SCNCs suspension with a consistency of $0.2 \%$ was cast in polystyrene Petri dishes at $50{ }^{\circ} \mathrm{C}$. After conditioned for $24 \mathrm{~h}$, the films were cut into strips with a length of $35 \mathrm{~mm}$ and a width of $15 \mathrm{~mm}$. The thickness of strips was measured with L\&W micrometer (Lorentzen \& Wettre, Stockholm, Sweden). The tests to determine the mechanical properties were performed with a tensile and compression tester (Instron 5565, Instron, Norwood, MA) equipped with a $500 \mathrm{~N}$ load cell, and a crosshead span of $20 \mathrm{~mm}$ and a strain rate of $4 \mathrm{~mm} / \mathrm{min}$ were set for the tests. The tensile strength, Young's modulus, and strain at break were recorded.

\section{Contact Angle Analysis of the Sulfonated Cellulose Nanocrystals (SCNCs) Films}

The hydrophilic property of the films was examined via a contact angle meter (SL200KB, Kino Industry Co., Ltd. Boston, MA). Deionized water was used as the probe liquid. A droplet of water $(2 \mu \mathrm{L})$ was dropped onto the film surface, the images of the droplet were captured with a digital camera, and the contact angle was automatically calculated with the drop shape analysis system CAST 3.0 (Kino Industry Co. Ltd. Boston, MA).

\section{RESULTS AND DISCUSSION}

\section{Oxidation and Sulfonation of the Microcrystalline Cellulose (MCC)}

In this study, cellulose nanocrystals were separated from MCC via successive periodate oxidation and bisulfite sulfonation. The MCC was first oxidized via sodium periodate to form DAC with an aldehyde groups content of $4.32 \mathrm{mmol} / \mathrm{g}$. Metallic salts and an elevated temperature can accelerate the oxidation reaction; thus, a higher aldehyde content can be achieved in comparison to an oxidation process at room temperature without the addition of salts (Sirviö et al. 2011). However, including metallic salts and having a higher temperature promotes DAC chain breakdown and increases the solubility of DAC (Kim et al. 2004), which led to an oxidation yield of only $67.3 \%$.

A stable and homogenous suspension was obtained after the DAC samples were sulfonated for 6,12 , and $24 \mathrm{~h}$, respectively, and these nanocrystals suspensions were visually evaluated with the Tyndall effect, which refers to a bright light beam is visible as a beam of light passes through a colloid suspension (Voskoboinikov et al. 2011). There was weak Tyndall effect presented in the $\mathrm{SCNC}_{1}$ suspension, as shown in Fig.1 (B). In order to obtain the nanocrystals, the suspension was centrifuged twice, and the gel at the bottom of the tube was collected. After dilution, dialysis, centrifugation, and sonication, the collected gel became a stable, clear, and transparent suspension, and displayed the Tyndall effect, as shown in Fig. 1(D). The SCNCs yield from the MCC sulfonated for 6, 12 , and $24 \mathrm{~h}$ were $54.4 \%, 51.6 \%$, and $45.8 \%$ respectively, i.e., the longer the sulfonation, the greater the mass loss. The mass loss may be caused by the dissolution of the amorphous part during the sulfonation step, and the yield was lower than reported by Rajalaxmi et al. (2010) (the yield was $87 \%$ to $94 \%$ ). This was probably due to the lower aldehyde content of DAC $(0.28 \mathrm{mmol} / \mathrm{g})$, compared to the aldehyde content in this study $(4.32 \mathrm{mmol} / \mathrm{g})$. 
In addition, the suspension was difficult to filter. There was no filtrate at all when the suspension was filtered with a hydrophilic MCE membrane (a pore size of $0.65 \mu \mathrm{m}$ ) under a vacuum pressure of $0.08 \mathrm{MPa}$, which indicated that the sulfonated cellulose possessed a strong capability of absorbing water. Therefore, the suspension was purified via dialysis.

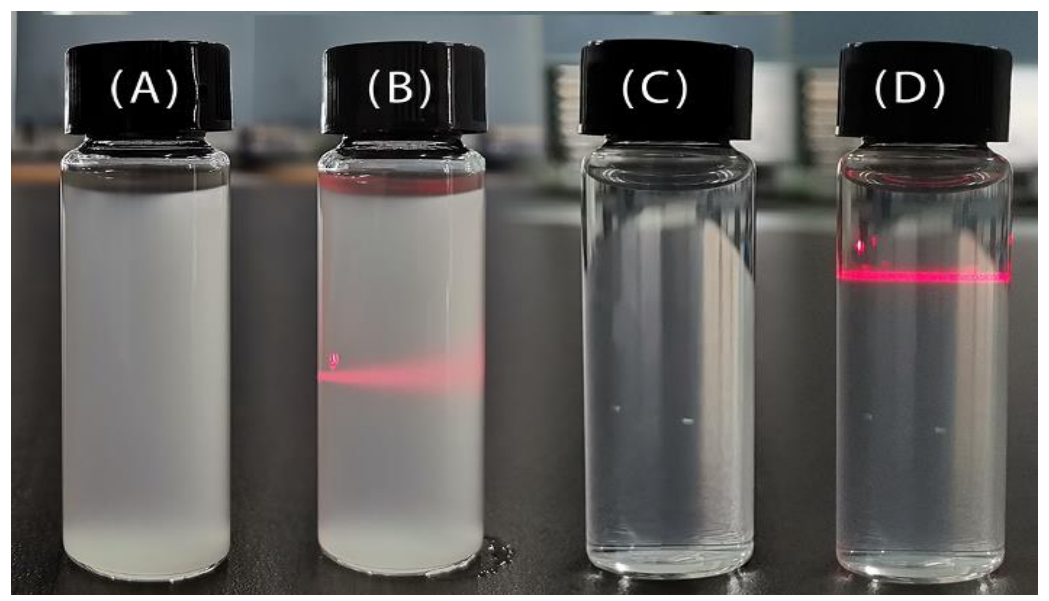

Fig. 1. Photographs of the $\mathrm{SCNC}_{1}$ suspensions without laser illumination $(\mathrm{A})$ and with laser illumination $(\mathrm{B})$; the $\mathrm{SCNC}_{1}$ suspension after sonication without laser illumination $(\mathrm{C})$ and with laser illumination (D)

\section{Fourier Transform Infrared (FTIR) Analysis}

The changes in the chemical structure were investigated via FTIR. The results are shown in Fig. 2. The weak peak at $1726 \mathrm{~cm}^{-1}$ was identified as the characteristic band of an aldehyde group, and the band at $891 \mathrm{~cm}^{-1}$ was attributed to hemiacetal and hydrate aldehyde (Speddin 1960; Sabzalian et al. 2014), which demonstrated the successful conversion of MCC into DAC. In the case of the SCNCs samples, the weak peaks at the $1160 \mathrm{~cm}^{-1}$ and $1115 \mathrm{~cm}^{-1}$ bands were regarded as symmetric and asymmetric of stretching of the $\mathrm{S}=\mathrm{O}$ bonds (Suganuma et al. 2008). However, the peaks at the $1726 \mathrm{~cm}^{-1}$ and 891 $\mathrm{cm}^{-1}$ bands appeared on all the sulfonated celluloses, which indicated that only a part of the aldehyde groups were converted into $\mathrm{SO}_{3}{ }^{-}$groups.

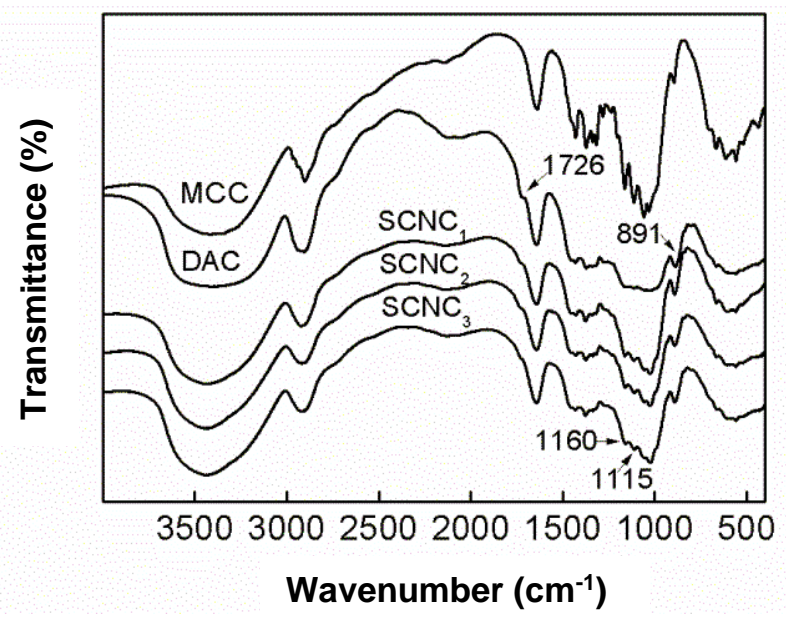

Fig. 2. FTIR spectra of the samples 


\section{Polyelectrate Titration}

The charge densities of the SCNCs were determined via polyelectrate titration. The charge densities of the $\mathrm{SCNC}_{1}, \mathrm{SCNC}_{2}$, and $\mathrm{SCNC}_{3}$ samples were found to be $-0.44,-0.57$, and $-0.75 \mathrm{meq} / \mathrm{g}$, respectively, which revealed that more aldehyde groups were converted into sulfonated groups with a longer sulfonation reaction. The negative sulfonated groups established an electrostatic repulsion effect between the SCNCs and played an important role in the stability of the sulfonated cellulose suspensions, which appeared clear, transparent, and homogenous even after 3 months of storage. The results indicated that a stable sulfonated cellulose suspensions could be obtained via the sulfonation of DAC at an equal dosage of $\mathrm{NaHSO}_{3}$ (mass ratio of $\mathrm{DAC}$ to $\mathrm{NaHSO}_{3}=1$ to 1 ) at room temperature for $6 \mathrm{~h}$.

\section{Morphology of the Sulfonated Cellulose Nanocrystals (SCNCs)}

Dialdehyde cellulose is insoluble in water due to the hemiacetals and acetyls in its cellulosic structure (Kim et al. 2004). However, the dissolution of some of the DAC amorphous regions enabled the liberation of $\mathrm{CNC}$ during the amination process of DAC (Sirviö et al. 2016). The mechanisms for the liberation of the SCNCs via the sulfonation of DAC may be the same as the DAC amination procedure. The morphologies of the $\mathrm{SCNC}_{2}$ and $\mathrm{SCNC}_{3}$ samples are presented in Fig. 3.

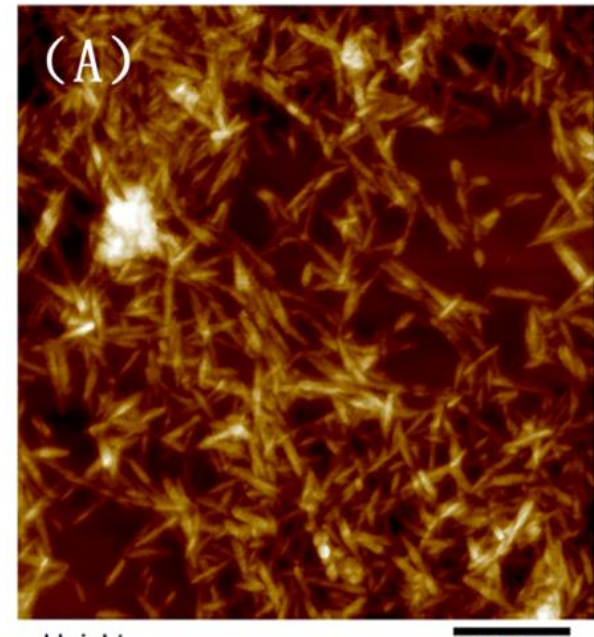

Height

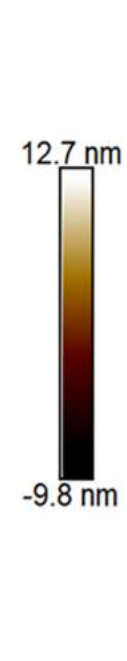

$\overline{300.0 \mathrm{~nm}}$

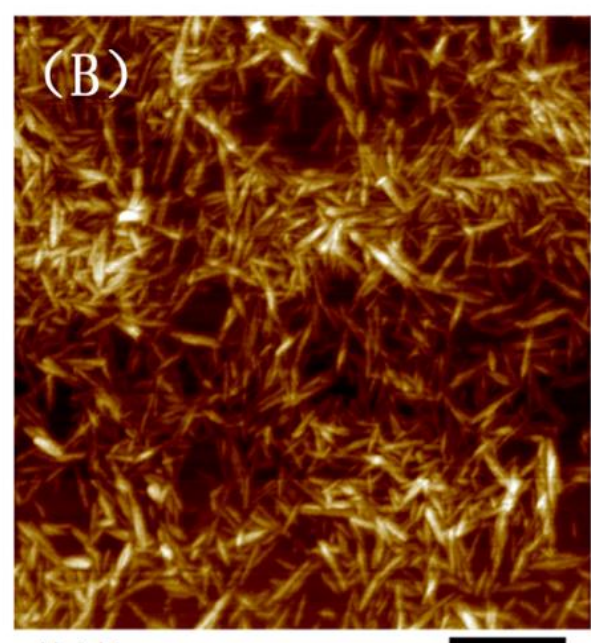

$\overline{300.0 \mathrm{~nm}}$

Fig. 3. AFM height images of $\mathrm{SCNC}_{2}(\mathrm{~A})$; and $\mathrm{SCNC}_{3}(\mathrm{~B})$

The SCNCs were rod-like and tended to aggregate. The average length of the $\mathrm{SCNC}_{2}$ and $\mathrm{SCNC}_{3}$ nanoparticles was $152.5 \mathrm{~nm} \pm 55.9 \mathrm{~nm}$ and $142 \mathrm{~nm} \pm 29.4 \mathrm{~nm}$, respectively, and the average width was $9.47 \mathrm{~nm} \pm 1.70 \mathrm{~nm}$ and $8.68 \mathrm{~nm} \pm 1.43 \mathrm{~nm}$, respectively. Both the morphology and the dimensions of the SCNCs were similar to the morphology and the dimensions of the CNC samples derived via sulfuric acid hydrolysis (Dong et al. 1998) and the morphology and the dimensions of the CNC derived via a successive periodate oxidation and heating treatment (Yang et al. 2015).

\section{Particle Size Analysis}

The size of the SCNCs was also determined via dynamic light scattering (DLS) and the results are presented in Fig. 4. The Z-average size of the $\mathrm{SCNC}_{1}, \mathrm{SCNC}_{2}$, and $\mathrm{SCNC}_{3}$ 
samples were $266.1 \mathrm{~nm} \pm 14 \mathrm{~nm}, 168.2 \mathrm{~nm} \pm 4.5 \mathrm{~nm}$, and $111.1 \mathrm{~nm} \pm 4.1 \mathrm{~nm}$, respectively, which were close to size of the nanocrystals extracted via successive periodate and chlorite oxidation (Yang et al. 2013). The results indicated that the longer the sulfonation reaction, the smaller the SCNCs particles. The polydispersity indexes of the $\mathrm{SCNC}_{1}, \mathrm{SCNC}_{2}$, and $\mathrm{SCNC}_{3}$ samples were $0.434 \pm 0.05,0.434 \pm 0.02$, and $0.412 \pm 0.08$, which revealed that the longer the sulfonation reaction, the more uniform the dimensions of the SCNCs.

In addition, only one peak was recorded in the DLS profiles for all SCNCs samples, whereas the acid hydrolysis subjected CNCs exhibited two peaks due to the orientation of rod-shaped CNCs (Shanmugarajah et al. 2015). One peak distribution possibly originated from the aggregation of the SCNCs. The DLS profile of the CNC separated via successive periodate and $\mathrm{NaBH}_{4}$ reduction also showed a one peak distribution (Errokh et al. 2018). Both the DLS and AFM results demonstrated that the dimensions of the SCNCs tended to become smaller as the sulfonation reaction time increased.

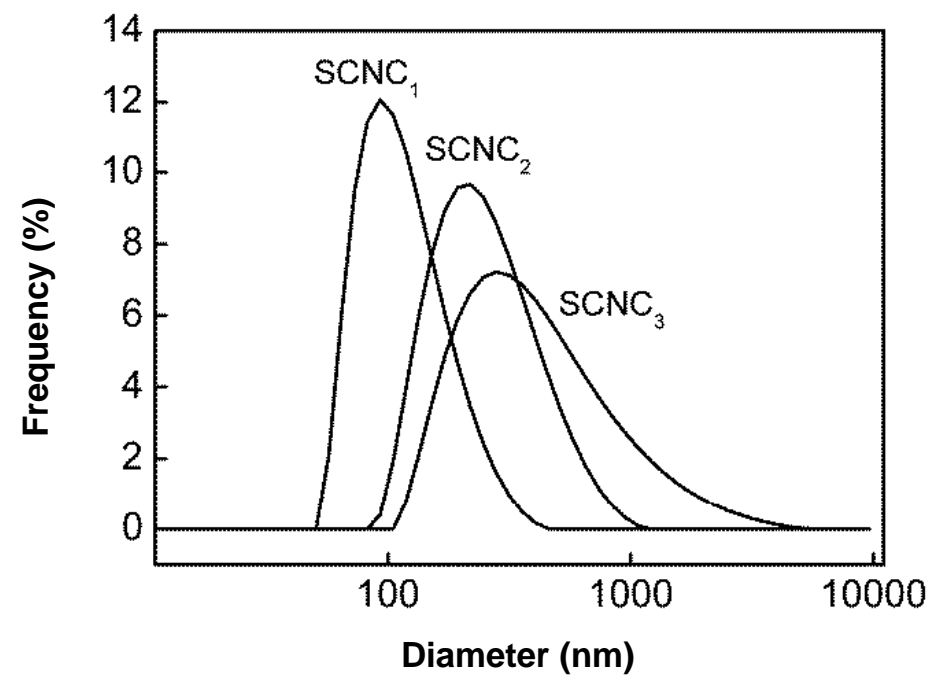

Fig. 4. Size and distribution of the SCNCs particles determined via DLS

\section{Determination of the Crystalline Index via X-Ray Diffraction (XRD)}

The XRD profiles of the sulfonated cellulose (SC) samples are presented in Fig. 5. All the diffractograms exhibited typical peaks at $14.5^{\circ}, 16.5^{\circ}$, and $22.6^{\circ}$, which corresponded to Bragg angles of $1 \overline{1} 0,110$, and 200 crystalline planes respectively. This indicated that the SCNCs had the same polymorphs as cellulose I (Sirviö et al. 2011; Yang et al. 2013; Sun et al. 2015). When the MCC was oxidized with periodate, the glucopyranose rings opened and the ordered structures were destroyed, which led to a decrease in the CI, from $82.5 \%$ for MCC to $41.6 \%$ for DAC, which was in agreement with the report by Kim et al. (2000).

In the sulfonation step, the hemiacetal bonds were disrupted, and the amorphous parts of the cellulose were dissolved. Therefore, the CI of the $\mathrm{SCNC}_{1}, \mathrm{SCNC}_{2}$, and $\mathrm{SCNC}_{3}$ samples increased to $56.4 \%, 62.6 \%$, and $64.1 \%$, respectively, which was confirmed by the decrease in the yield of the sulfonation reaction. The longer the sulfonation reaction, the more the amorphous parts were dissolved and the higher the CI of the SCNCs. Errokh et al. (2018) also reported an increase in CI during the $\mathrm{NaBH}_{4}$ reduction of DAC. 


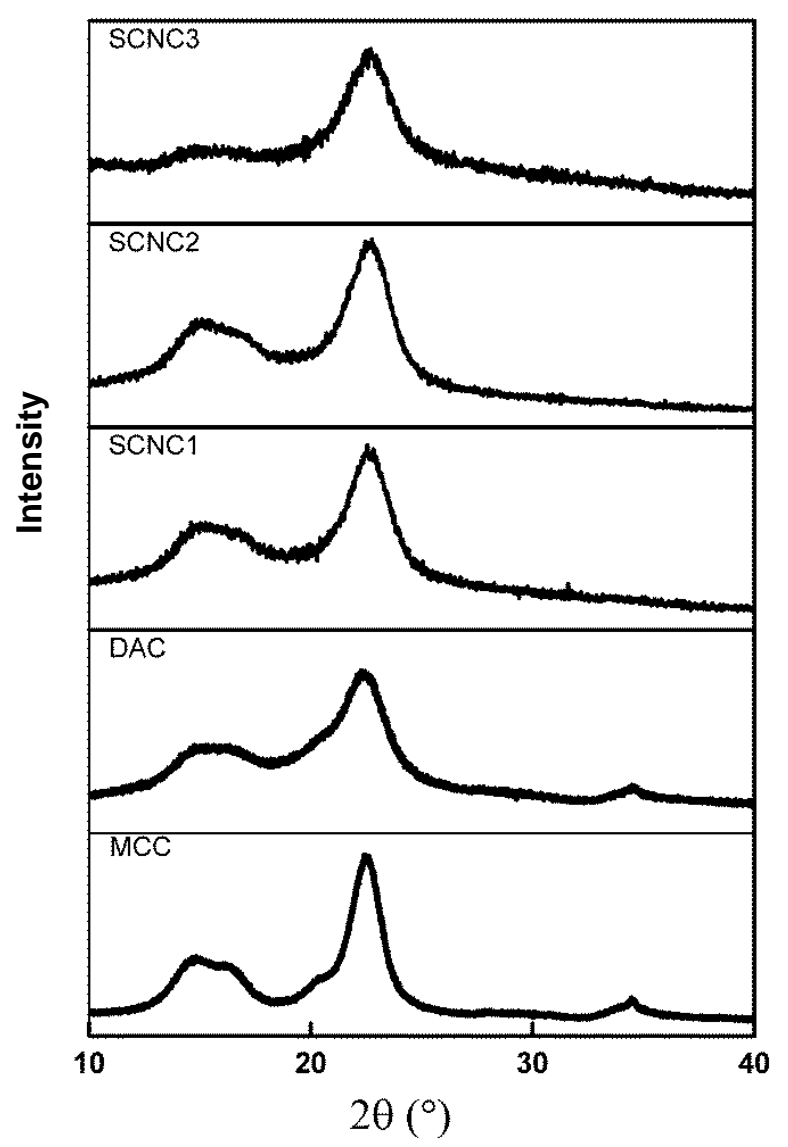

Fig. 5. The diffractograms of the SCNCs

\section{Mechanical Strength of the Films}

Highly transparent films were obtained via the casting of the SCNCs suspensions, and the films are shown in Fig. 6. The thickness of these films ranged from $30 \mu \mathrm{m}$ to 45 $\mu \mathrm{m}$. The films cast from $\mathrm{SCNC}_{2}$ and $\mathrm{SCNC}_{3}$ were so brittle that cracks appeared at the edges of the films when they were cut into strips, which may be ascribed to a higher crystallinity index $\left(56.4 \%\right.$ for $\mathrm{SCNC}_{1}, 62.6 \%$ for $\mathrm{SCNC}_{2}$ and $64.1 \%$ for $\left.\mathrm{SCNC}_{3}\right)$ and a relatively small size of the nanocrystals (Z-average size of $\mathrm{SCNC}_{1}, \mathrm{SCNC}_{2}$, and $\mathrm{SCNC}_{3}$ were $266.1,168.2$, and $111.1 \mathrm{~nm}$ ), so only the mechanical properties of the $\mathrm{SCNC}_{1}$ films were obtained. The Young's modulus, tensile strength, and strain at break of the $\mathrm{SCNC}_{1}$ film were $4.12 \mathrm{GPa} \pm 0.43 \mathrm{GPa}, 49 \mathrm{MPa} \pm 5 \mathrm{MPa}$, and $1.69 \% \pm 0.14 \%$, respectively, as shown in Fig. 7. Visanko et al. (2015) extracted CNC (ADCNC) with an aspect ratio of 50 via the amination of DAC, and Bras et al. (2011) extracted CNC (AHCNC) with an aspect ratio of 11.3 via acid hydrolysis. The Young's modulus of the ADCNC and AHCNC films were 5.7 and $2.14 \mathrm{GPa}$, respectively. The SCNCs in this paper were rigid and rod-like, which was the same as the ADCNC and AHCNC. For the rigid and rod-like CNC, the aspect ratio plays an important role in terms of Young's modulus; the higher the aspect ratio, the higher the Young's modulus (Bras et al. 2011). The Young's modulus of the SCNCs film was lower than the Young's modulus of the ADCNC film, which was due to the lower aspect ratio of the SCNCs (16.7). The aspect ratio of the SCNCs and AHCNC 
were similar; the higher Young's modulus of the SCNCs film was ascribed to hemiacetyl cross-linking and a higher aspect ratio. Liimatainen and Visanko (2013) prepared CNF, with a width of 10 to $60 \mathrm{~nm}$ and a length of several micrometers, via the sulfonation of DAC followed by homogenization. This CNF film had a Young's modulus of $13.5 \mathrm{GPa}$, which was much higher than the Young's modulus of the $\mathrm{SCNC}_{1}$ film. The CNF was flexible and had a large aspect ratio and was able to entangle each other during the process of film formation, so the CNF films had a higher Young's modulus than the $\mathrm{SCNC}_{1}$ film.
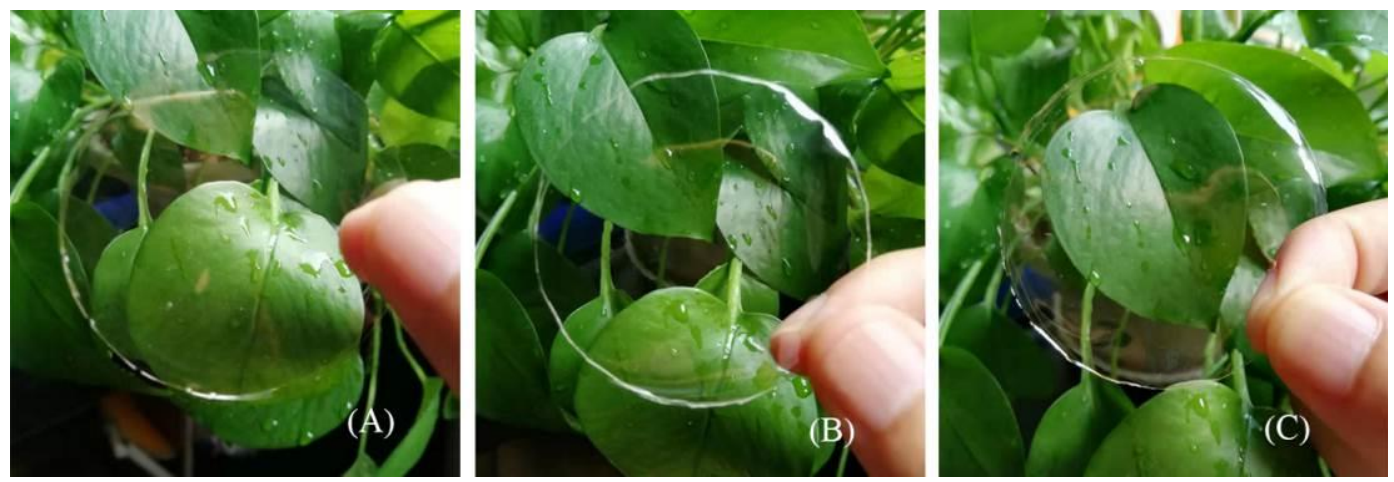

Fig. 6. Appearance of the transparent $\mathrm{SCNC}_{1}$ film (A); $\mathrm{SCNC}_{2}$ film (B); and $\mathrm{SCNC}_{3}$ film (C)

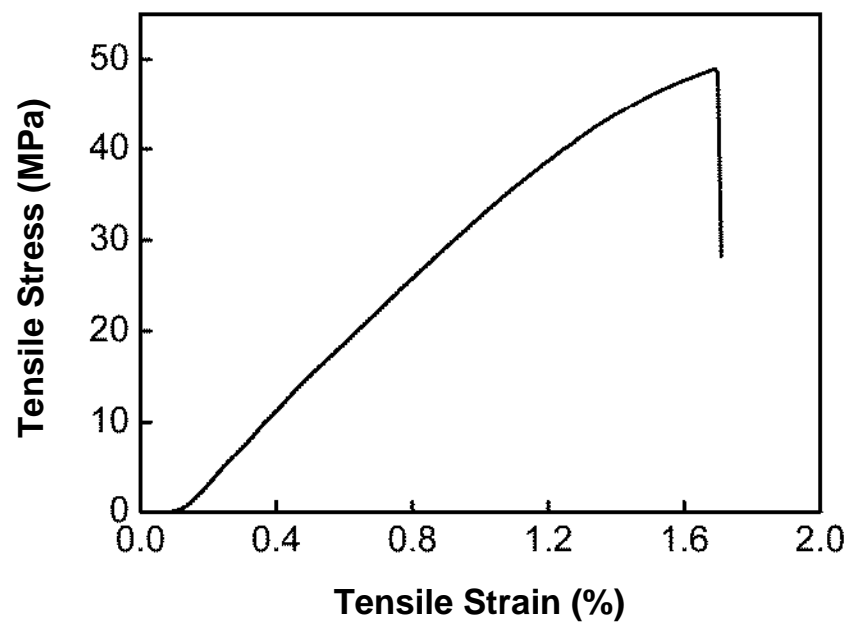

Fig 7. Stress vs. strain of the $\mathrm{SCNC}_{1}$ film

\section{Hydrophilicity of the Films}

The hydrophilic property of the SCNCs films was evaluated via dynamic contact angle tests, and the results are presented in Fig. 8. The initial contact angles of the $\mathrm{SCNC}_{1}$, $\mathrm{SCNC}_{2}$, and $\mathrm{SCNC}_{3}$ samples were $33.7^{\circ} \pm 5.1^{\circ}, 30.63^{\circ} \pm 4.3^{\circ}$, and $27.4^{\circ} \pm 4.8^{\circ}$, respectively. Compared with DCC, which had a contact angle of $45^{\circ}$ (Visanko et al. 2014), and TEMPO nanofibrils, which had a contact angle of $52^{\circ}$ (Rodionova et al. 2012), the SCNCs films had the lowest contact angle. The value of the contact angle of the films decreased as the sulfonated group content increased. The results indicated that the SCNCs were highly hydrophilic, and the hydrophilicity was ascribed to a large number of hydroxyl and sulfonated groups on the surface of the SCNCs. 


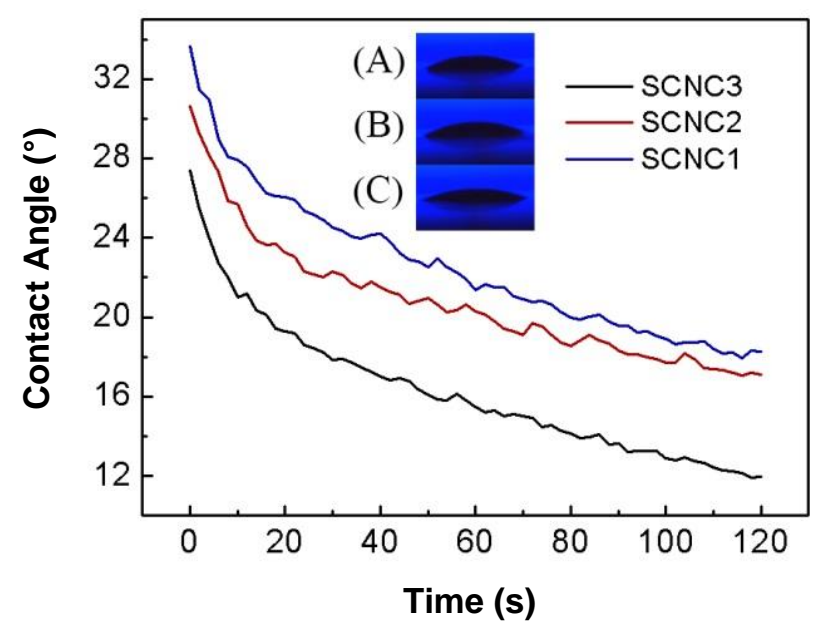

Fig. 8. Dynamic contact angle plot of SCNCs films and photographs of the initial contact angle of $\operatorname{SCNC}_{1}(\mathrm{~A}), \mathrm{SCNC}_{2}(\mathrm{~B})$ and $\mathrm{SCNC}_{3}(\mathrm{C})$

\section{CONCLUSIONS}

1. Microcrystalline cellulose was oxidized with sodium periodate followed by sulfonation with sodium bisulfite, and a stable, transparent, and homogenous sulfonated cellulose suspension was obtained.

2. The rod-like sulfonated cellulose nanocrystals ( $\mathrm{SCNCs}$ ), which had an average length of $140 \mathrm{~nm}$ to $153 \mathrm{~nm}$ and an average width of 8 to $10 \mathrm{~nm}$, were extracted via dialysis, centrifugation, and sonication of the sulfonated cellulose suspension.

3. Compared with dialdehyde cellulose (DAC), the SCNCs had a higher crystalline index, which ranged from $56 \%$ to $64 \%$.

4. The SCNCs films are transparent and had a Young's modulus of $4.12 \mathrm{GPa}$ and a tensile strength of $49 \mathrm{MPa}$. In addition, the SCNCs film was highly hydrophilic and the contact angle of the SCNCs films reached a minimum of $27.4^{\circ}$

\section{ACKNOWLEDGMENTS}

The authors sincerely acknowledge and appreciate the funding support by the Guangdong Industry Polytechnic Research Team Program (Grant No. KYTD2018-005) and the Guangdong Industry Polytechnic Natural Science Foundation Program (Grant No. KYTD2019-008)

\section{REFERENCES CITED}

Bras, J., Viet, D., Bruzzese, C., and Dufresne, A. (2011). "Correlation between stiffness of sheets prepared from cellulose whiskers and nanoparticles dimensions,"

Carbohydrate Polymers 84(1), 211-215. DOI: 10.1016/j.carbpol.2010.11.022 
Casu, B., Naggi, A., Torri, G., Allegra, G., Meille, S. V., Cosani, A., and Terbojevich, M. (1985). "Stereoregular acyclic polyalcohols and polyacetates from cellulose and amylose," Macromolecules 18(12), 2762-2767. DOI: 10.1021/ma00154a068

Dong, X. M., Revol, J.-F., and Gray, D. G. (1998). "Effect of microcrystallite preparation conditions on the formation of colloid crystals of cellulose," Cellulose 5(1), 19-32. DOI: 10.1023/A:1009260511939

Errokh, A., Magnin, A., Putaux, J.-L., and Boufi, S. (2018). "Morphology of the nanocellulose produced by periodate oxidation and reductive treatment of cellulose fibers," Cellulose 25(7), 3899-3911. DOI: 10.1007/s10570-018-1871-7

Goetz, L., Mathew, A., Oksman, K., Gatenholm, P., and Ragauskas, A. J. (2009). "A novel nanocomposite film prepared from crosslinked cellulosic whiskers," Carbohydrate Polymers 75(1), 85-89. DOI: 10.1016/j.carbpol.2008.06.017

Guigo, N., Mazeau, K., Putaux, J.-L., and Heux, L. (2014). "Surface modification of cellulose microfibrils by periodate oxidation and subsequent reductive amination with benzylamine: a topochemical study," Cellulose 21(6), 4119-4133.

DOI: $10.1007 / \mathrm{s} 10570-014-0459-0$

Kim, U.-J., Kuga, S., Wada, M., Okano, T., and Kondo, T. (2000). "Periodate oxidation of crystalline cellulose," Biomacromolecules 1(3), 488-492.

DOI: $10.1021 / \mathrm{bm} 0000337$

Kim, U.-J., Wada, M., and Kuga, S. (2004). "Solubilization of dialdehyde cellulose by hot water," Carbohydrate Polymers 56(1), 7-10. DOI: 10.1016/j.carbpol.2003.10.013

Klemm, D., Heublein, B., Fink, H.-P., and Bohn, A. (2005). "Cellulose: Fascinating biopolymer and sustainable raw material," Angewandte Chemie International Edition 44(22), 3358-3393. DOI: 10.1002/anie.200460587

Liimatainen, H., Visanko, M., Sirviö, J., Hormi, O., and Niinimäki, J. (2013). "Sulfonated cellulose nanofibrils obtained from wood pulp through regioselective oxidative bisulfite pre-treatment," Cellulose, 20(2), 741-749. DOI: 10.1007/s10570013-9865-y

Nechyporchuk, O., Belgacem, M. N., and Bras, J. (2016). "Production of cellulose nanofibrils: A review of recent advances," Industrial Crops and Products 93, 2-25. DOI: 10.1016/j.indcrop.2016.02.016

Pan, S., and Ragauskas, A. J. (2014). "Enhancement of nanofibrillation of softwood cellulosic fibers by oxidation and sulfonation," Carbohydrate Polymers 111, 514-523. DOI: 10.1016/j.carbpol.2014.04.096

Rajalaxmi, D., Jiang, N., Leslie, G., and Ragauskas, A. J. (2010). "Synthesis of novel water-soluble sulfonated cellulose," Carbohydrate Research 345(2), 284-290. DOI: 10.1016/j.carres.2009.09.037

Rånby, B. G. (1949). “Aqueous colloidal solutions of cellulose micelles," Acta Chemica Scandinavica 3, 649-650. DOI: 10.3891/acta.chem.scand.03-0649

Rocha, I., Ferraz, N., Mihranyan, A., Strømme, M., and Lindh, J. (2018). "Sulfonated nanocellulose beads as potential immunosorbents," Cellulose, 25(3), 1899-1910. DOI: $10.1007 / \mathrm{s} 10570-018-1661-2$

Rodionova, G., Eriksen, Ø., and Gregersen, Ø. (2012). “TEMPO-oxidized cellulose nanofiber films: Effect of surface morphology on water resistance," Cellulose 19(4), 1115-1123. DOI: $10.1007 / \mathrm{s} 10570-012-9721-5$

Sabzalian, Z., Alam, N., and van de Ven, T. G. M. (2014). "Hydrophobization and characterization of internally crosslink-reinforced cellulose fibers," Cellulose 21(3), 1381-1393. DOI: 10.1007/s10570-014-0178-6 
Segal, L., Creely, J. J., Martin, A. E., and Conrad, C. M. (1959). “An empirical method for estimating the degree of crystallinity of native cellulose using the X-Ray diffractometer," Textile Research Journal 29(10), 786-794.

DOI: $10.1177 / 004051755902901003$

Shanmugarajah, B., Kiew, P. L., Chew, I. M. L., Choong, T. S. Y., and Tan, K. W. (2015). "Isolation of nanocrystalline cellulose (NCC) from palm oil empty fruit bunch (EFB): Preliminary result on FTIR and DLS analysis," Chemical Engineering Transactions 45, 1705-1710. DOI: 10.3303/CET1545285

Sirviö, J. A., Visanko, M., Laitinen, O., Ämmälä, A., and Liimatainen, H. (2016). "Amino-modified cellulose nanocrystals with adjustable hydrophobicity from combined regioselective oxidation and reductive amination," Carbohydrate Polymers 136, 581-587. DOI: 10.1016/j.carbpol.2015.09.089

Sirviö, J., Hyvakko, U., Liimatainen, H., Niinimaki, J., and Hormi, O. (2011). "Periodate oxidation of cellulose at elevated temperatures using metal salts as cellulose activators," Carbohydrate Polymers 83(3), 1293-1297.

DOI: 10.1016/j.carbpol.2010.09.036

Sirviö, J., Liimatainen, H., Niinimäki, J., and Hormi, O. (2011). "Dialdehyde cellulose microfibers generated from wood pulp by milling-induced periodate oxidation," Carbohydrate Polymers 86(1), 260-265. DOI: 10.1016/j.carbpol.2011.04.054

Speddin, H. (1960). "Infrared spectra of periodate-oxidised cellulose," Journal of the Chemical Society 73, 3147-3152. DOI: 10.1039/jr9600003147

Suganuma, S., Nakajima, K., Kitano, M., Yamaguchi, D., Kato, H., Hayashi, S., and Hara, M. (2008). "Hydrolysis of cellulose by amorphous carbon bearing $\mathrm{SO}_{3} \mathrm{H}$, $\mathrm{COOH}$, and $\mathrm{OH}$ groups," Journal of the American Chemical Society 130(38), 12787 12793. DOI: 10.1021/ja803983h

Sun, B., Hou, Q., Liu, Z., and Ni, Y. (2015). "Sodium periodate oxidation of cellulose nanocrystal and its application as a paper wet strength additive," Cellulose 22(2), 1135-1146. DOI: $10.1007 / \mathrm{s} 10570-015-0575-5$

Sun, F., Liu, W., Dong, Z., and Deng, Y. (2017). “Underwater superoleophobicity cellulose nanofibril aerogel through regioselective sulfonation for oil/water separation," Chemical Engineering Journal 330, 774-782.

DOI: 10.1016/j.cej.2017.07.142

Thiangtham, S., Runt, J., and Manuspiya, H. (2019). "Sulfonation of dialdehyde cellulose extracted from sugarcane bagasse for synergistically enhanced water solubility," Carbohydrate Polymers 208, 314-322. DOI: 10.1016/j.carbpol.2018.12.080

Visanko, M., Liimatainen, H., Sirviö, J. A., Heiskanen, J. P., Niinimäki, J., and Hormi, O. (2014). "Amphiphilic cellulose nanocrystals from acid-free oxidative treatment: Physicochemical characteristics and use as an oil-water stabilizer," Biomacromolecules 15(7), 2769-2775. DOI: 10.1021/bm500628g

Voskoboinikov, I. V., Konstantinova, S. A., Korotkov, A. N., and Gal'braikh, L. S. (2011). "Process for preparing nanocrystalline cellulose," Fibre Chemistry 43(2), 125-128. DOI: 10.1007/s10692-011-9318-z

Yang, H., Alam, N., and van de Ven, T. G. M. (2013). "Highly charged nanocrystalline cellulose and dicarboxylated cellulose from periodate and chlorite oxidized cellulose fibers," Cellulose 20(4), 1865-1875. DOI: 10.1007/s10570-013-9966-7

Yang, H., Chen, D., and van de Ven, T. G. M. (2015). "Preparation and characterization of sterically stabilized nanocrystalline cellulose obtained by periodate oxidation of cellulose fibers," Cellulose 22(3), 1743-1752. DOI: 10.1007/s10570-015-0584-4 
Zhao, H., and Heindel, N. D. (1991). "Determination of degree of substitution of formyl groups in polyaldehyde dextran by the hydroxylamine hydrochloride method," Pharmaceutical Research 8(3), 400-402. DOI: 10.1023/A:1015866104055

Article submitted: October 21, 2020; Peer review completed: December 27, 2020;

Revised version received and accepted: January 19, 2021; Published: January 21, 2021.

DOI: $10.15376 /$ biores.16.1.1713-1725 